This is the preprint version of the article published as

A. Layek, J. Van Loon, M.B.J. Roeffaers, A.V. Kubarev, Catal. Sci. Technol., 2019, DOI: 10.1039/c9cy00948e.

\title{
ARTICLE
}

Received 00th January 20xx, Accepted 00th January 20xx DOI: $10.1039 / \times 0 \times x 00000 x$

\section{Correlated Super-Resolution Fluorescence and Electron Microscopy Reveals the Catalytically Active Nanorods within Individual H-ZSM-22 Zeolite Particles}

\author{
Arunasish Layek, ${ }^{\# \text { a }}$ Jordi Van Loon, ${ }^{\# a}$ Maarten B.J. Roeffaers, ${ }^{\mathrm{a}}$ and Alexey V. Kubareva
}

\begin{abstract}
Nanocrystalline zeolite particles are applied in a wide range of catalytic reactions. Their size, shape, and the distribution of catalytically active sites, significantly varies throughout zeolite batches and within individual particles. This variability leads to a heterogeneous distribution of catalyst performance. Directly investigating the structure-activity relationship at the nanoscale is essential for the rational improvement of catalyst materials. In this work, integrated fluorescence and electron microscope is employed to correlatively study the structure and performance of individual H-ZSM-22 particles. The needleshaped morphology of these zeolite particles originates from the lateral fusion of multiple elementary nanorods. Indirect bulk scale experiments have suggested that this process converts catalytically inactive external Al into catalytically active internal Al. The correlative investigation performed in this research provides direct evidence that this conversion takes place, as an inactive shell of $20-40 \mathrm{~nm}$ thickness is observed and reactivity is confined to the crystal core. Furthermore, within the catalyst particles nanometer scale catalytic hotspots have been revealed and they are assumed to result from the presence of structural imperfections that locally increase accessibility into the microporous structure. Linear polarized excitation light experiments confirmed that catalytic transformations exclusively occurred on acid sites confined within the microporous structure.
\end{abstract}

\section{Introduction}

Zeolites are crystalline microporous aluminosilicates extensively used as heterogeneous catalysts in (petro)chemical and environmental industries. ${ }^{[1-4]}$ The acid sites confined within the microporous structure of the zeolite are decisive for many catalytic applications. The number of potential catalytically active acid sites is indicated by the framework Si/Al ratio, as every replacement of a framework $\mathrm{Si}$ atom by an $\mathrm{Al}$ atom can generate a strong Brønsted acid bridging hydroxyl group. The strength of these Brønsted acid sites depends on the spatial distribution of $\mathrm{Al}$ atoms throughout the zeolite crystals. Additionally, the position of the Al atom in the framework is also closely related to acid site accessibility and the confinement of reacting molecules inside the microporous structure. Such confinement often leads to the essential shape selective properties, which zeolites are known for. However, mass transport towards the active sites within catalyst particles is typically limited due to this tight confinement. This hampers catalyst performance, which is governed by an intricate interplay of multiple factors. ${ }^{[5-7]}$

Centre for Membrane Separations, Adsorption, Catalysis and Spectroscopy for Sustainable Solutions, Department of Microbial and Molecular Systems, KU Leuven, Celestijnenlaan 200F, 3001 Leuven, Belgium.

E-mail: marten.roeffaers@kuleuven.be

\# These authors contributed equally to this work.

$\S$ Current address: Adamas Knowledge City, Department of Chemistry, Adamas University, Barasat - Barrackpore Rd, Kolkata, West Bengal 700126, India.

${ }^{\dagger}$ Electronic Supplementary Information (ESI) available. See DOI: 10.1039/x0xx00000x
H-ZSM-22 is a high silica zeolite of the TON framework-type consisting of 5-, 6-, and 10-membered ring channels. ${ }^{[5,8]}$ The latter are oriented along the crystallographic c-axis and constitute a unidirectional porous structure inside the H-ZSM22 crystals, as only their ca. $0.46 \times 0.57 \mathrm{~nm}^{2}$ dimension are sufficiently large to enable considerable mass transport of organic molecules. ${ }^{[9-12]}$ Typically, $\mathrm{H}-\mathrm{ZSM}-22$ particles have been reported to grow as individual rod-like crystals, i.e., the 'nanorods', which undergo lateral fusion during the late stages of the synthesis, leading to the typical needle-shaped aggregates (Figure 1). ${ }^{[13,14]}$

(A)

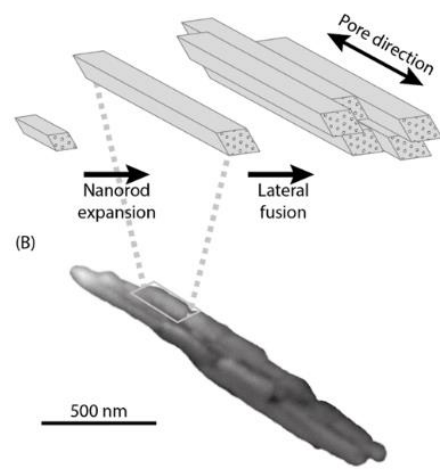


Figure 1. (A) Schematic representation of the three stages that occur during $\mathrm{H}$-ZSM-22 synthesis; nanorod formation, nanorod expansion and needle formation through lateral nanorod fusion. The direction of the one dimensional porous structure is indicated by the double headed arrow and the crystal faces where the pore openings are located are represented by the dotted planes. (B) A cropped-out SEM image of a typical needle-shaped H-ZSM-22 particle. Rectangle highlights an individual nanorod that is part of the larger aggregate.

The combination of a quasi-one-dimensional medium sized pore system and nanometer-scale particle sizes makes H-ZSM22 a promising shape-selective acid catalyst in processes such as the dewaxing of petroleum feedstocks, ${ }^{115,16]}$ (hydro)isomerization of hydrocarbons, ${ }^{[9,17-19]}$ naphtha cracking, ${ }^{[20]}$ methanol to light olefins conversion, ${ }^{[11,21,22]}$ dehydration of glycerol to acrolein, [23] etc.

The growth mechanism of needle-shaped H-ZSM-22 particles has been described in literature by several groups independently. ${ }^{[13,24,25]}$ Initially, amorphous particles are formed by the coalescence of silica sol grains around which a template (diethanolamine)-rich secondary gel precipitates. During synthesis, the silica rich core slowly dissolves while heterogeneous zeolite nucleation takes place at the organophilic surface of the secondary gel. These zeolite crystals are initially silicon rich, but in the later stages of zeolite growth Al is preferentially incorporated because of the accumulation of aluminate in the mother liquor. As a result, elementary nanorod particles are obtained with an Al enriched outer surface. This is indirectly confirmed by combined inductively coupled plasma atomic emission spectroscopy (ICP-AES) and X-ray photoelectron spectroscopy (XPS), ${ }^{[13,24]}$ while the possibility of direct $\mathrm{Al}$ concentration mapping by energy-dispersive X-ray spectrometry high-angle annular dark field scanning transmission electron microscopy (EDX-HAADF-STEM) is limited due to low Al content (Figure S1). Ex-situ scanning electron microscopy (SEM), transmission electron microscopy (TEM) and $\mathrm{X}$-ray diffraction (XRD) show that in the final stages of $\mathrm{H}$-ZSM22 synthesis these nanorods fuse together laterally and give rise to the larger needle-shaped aggregates. This lateral fusion, observed as a stepwise increase of the particle dimensions, is not correlated to a gain in zeolite yield. It can therefore not be attributed to simple crystal growth. Importantly, this nanorod fusion and increased H-ZSM-22 particle dimensions lead to a significant increase in catalytic activity which has been attributed to the conversion of inactive external $\mathrm{Al}$ into catalytically active internal Al.[13] Figure $1 \mathrm{~A}$ schematically represents this needle-shaped $\mathrm{H}$-ZSM-22 particle growth.

Individual nanorods with an average diameter of approximately $46 \mathrm{~nm}$, as determined by SEM on 23 different nanorods, are aggregated into needle-shaped crystals and their interfaces are assumed to be catalytically active. However, the experimental confirmation of such catalytic activity is so far only based on indirect evidence, as the intrinsic structural heterogeneity within solid catalytic systems inevitably results in intra- and inter-particle variances in catalytic activity. ${ }^{[26-29]}$ These differences cannot be straightforwardly revealed using traditional catalyst characterization techniques and exact information on the relationship between catalyst performance and its structure is therefore yet to be obtained. Moreover, the inactive external surface acid sites along with the constrained pore channel system in H-ZSM-22 would further enhance the shape selectivity by avoiding undesirable reactions. Hence, a quantitative technique to establish a correlation between the catalytic performance and the local nanocrystalline zeolite structure would be desirable. Such a detailed understanding of the structure-activity relationship combined with advanced synthetic and post-synthetic approaches could ultimately lead to an optimized application of H-ZSM-22 catalysts.

Super-resolution fluorescence microscopy has recently emerged as an effective approach to investigate solid acid catalysts at the single catalyst particle and even single catalytic turnover level.[27,29-31] For example, the technique called nanometer accuracy by stochastic chemical reactions (NASCA) microscopy works by adding a specific non-fluorescent fluorogenic reagent to the zeolite sample which is converted into a fluorescent reaction product at the catalytically active sites. ${ }^{[32]}$ By detecting these individual catalytic conversions and localizing their positions with nanometer accuracy, highly precise activity maps of individual catalyst particles can be reconstructed by accumulating the obtained data. One problem that remains is the correlation of these activity maps to the underlying nanoscale structural context that remains unresolved. The latter can be obtained by electron microscopy. For example, SEM offers highly resolved structural imaging capabilities to visualize nanoscale structural features and outer particle surfaces. By applying both NASCA and SEM in a combined approach, correlative chemical reactivity mapping and structural imaging of zeolite catalysts would enable a direct visualization of the structure-activity relationship within individual catalysts particles. ${ }^{[33]}$ In this work, we investigate the catalytic performance of needle-shaped $\mathrm{H}-\mathrm{ZSM}-22$ (Si/Al=75) particles by correlative NASCA and SEM. The highest catalytic activity is revealed in the center of the needle-shaped H-ZSM22 particles, whereas the rim of the particles shows significantly less activity. Moreover, sharp particle tips show either no or very low activity compared to thicker, blunt tips, which are catalytically active. These observations confirm earlier reports based on indirect experiments. Indeed, external Al does not contribute to the catalytic activity as opposed to internal Al. The catalytic activity screening of individual catalyst particles has furthermore revealed nanometer scale catalytic hotspots within the particles. These are attributed to an inhomogeneous active site distribution and accessibility resulting from incomplete sideways nanorod fusion. Additionally, reactivity mapping using linear polarized excitation light confirms that catalytic transformations only take place on active sites located inside the pore channels.

\section{Results and Discussion}

The experiments described in this work are performed on an inhouse built integrated light and electron microscope (ILEM), which is described in detail in the Supporting Information (Figure S2). Importantly, this set-up was used in a correlative fashion, i.e., initially SEM is performed under high vacuum 
conditions and afterwards the system is brought to atmospheric pressure prior to the addition of the fluorogenic furfuryl alcohol (FFA) reagent solution used to perform the catalytic activity mapping on the same H-ZSM-22 crystals. This approach was used to investigate over 20 individual needle-shaped H-ZSM-22 particles. In Figure 2A-D the experimental results of four representative crystals are shown. The catalytic activity maps are built up by simply counting individual reaction turnovers in projected areas of $20 \times 20 \mathrm{~nm}^{2}$ during a 300 second experiment. The color scale indicates the local reactivity within these areas. A detailed procedure describing the conversion of single turnover numbers into catalytic reactivity is provided in the supporting information. In Figure $2 \mathrm{E}-\mathrm{H}$ the corresponding scanning electron micrographs are shown, which are furthermore accurately overlaid with the catalytic activity maps into correlative micrographs, as displayed in Figure 2I-L. A detailed description of this procedure is also provided in the supporting information.

The correlative micrographs reveal that the catalytic activity is heterogeneously distributed along the long axis of the needleshaped particles, with at least one zone per particle showing an increased catalytic activity; these zones will be further referred to as catalytic hotspots. These hotspots do not seem to be directly related to any structural feature observed in SEM. However, when taking a closer look at the correlated micrographs, a catalytic hotspots can be associated to an intergrown nanorod on top of the needle-shaped H-ZSM-22 particle and other structural imperfections; see white arrows in the SEM images (Figure $2 \mathrm{E}-\mathrm{G}$ ).

Also across the width of the needle-shaped particles, the catalytic activity seems to be irregular. In general, the catalytic activity is low along the edges and reaches a maximum in the middle of the particle. Moreover, when comparing the width of the catalytic activity maps with the actual particle width obtained through SEM, a general discrepancy is often observed. For instance, the needle-shaped particle illustrated in Figure $2 \mathrm{E}$ has a physical width of approximately $160 \mathrm{~nm}$, whereas the catalytic activity map is only approximately $110 \mathrm{~nm}$ wide. This reveals a catalytically inactive zone of under $40 \mathrm{~nm}$ on both sides of $\mathrm{H}-\mathrm{ZSM}-22$ particles.
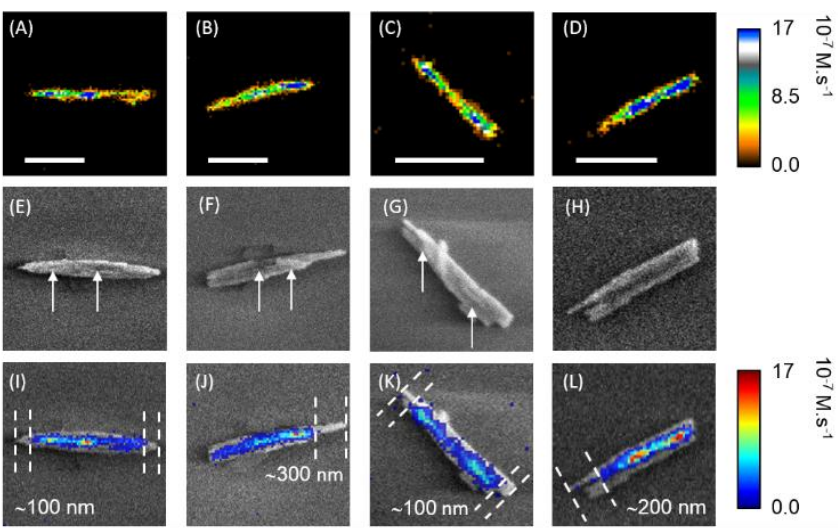

Figure 2. (A-D) Quantitative catalytic activity maps of four representative H-ZSM-22 crystals. The color bar indicates the reactivity within the individual bins $\left(20 \times 20 \mathrm{~nm}^{2}\right)$. $(\mathrm{E}-\mathrm{H})$ The respective SEM images of the four H-ZSM-22 crystallites and (I-L) the correlative micrographs including an alternative color scale that qualitatively represents the activity distribution. Dashed lines indicate the absence of catalytic activity in the tip zones of some crystals. Structural features that correspond to catalytic hotspots are additionally indicated by the white arrows. Scale bars $=500 \mathrm{~nm}$.

Note that also the external surface itself is found to be catalytically inactive. Additionally, a similar zone of inactivity is observed at the tips of the needle-shaped H-ZSM-22 particles, i.e., less activity is observed on the tips compared to the bulk of the particle. The dashed lines in Figure $2 \mathrm{I}-\mathrm{L}$ indicate these catalytically inactive areas.

The observations described above support the growth mechanism of needle-shaped H-ZSM-22 particles as proposed in literature (vide supra). ${ }^{[13]}$ More precisely, as lateral nanorod fusion is hypothesized to transform the catalytically inactive external Al into catalytically active internal $\mathrm{Al}$, a zone of inactivity at the edges of the needle-shaped crystals is to be expected. Moreover, the catalytic activity maps are a 2D projection of a 3D object, which is thicker in the center compared to the edges. Hence, more catalytically active sites due to internal $\mathrm{Al}$ are present at the center of the particles and this results in the locally increased number of turnovers. In Figure S5, the bright field transmission electron microscope (BFTEM) images and electron diffraction pattern are presented. The BF-TEM images revealed that the large needle-shaped $\mathrm{H}$ ZSM-22 particles are formed by stacking of single H-ZSM-22 nanorods (see Figure S5A-B). A representative single nanorod is depicted in Figure S5B which has a length of $170 \mathrm{~nm}$ and width of $30 \mathrm{~nm}$. An electron diffraction pattern in [0-11] zone axis orientation is depicted in Figure $\mathrm{S} 5 \mathrm{C}$, which proves the crystallinity of the sample. This observation suggests that very low activity at the exterior surfaces of the needle-shaped crystals is not due to amorphous zones, but rather due to catalytically inefficient external Al sites. The same rationale can also explain why sharp tips at the outer ends of the needleshaped H-ZSM-22 particles do not show significant catalytic activity, as opposed to blunt tips. Sharp tips are composed of individual nanorods which are only fused together close to one end along their longest dimension. Hence, the amount of catalytically active Al resulting from this fusion is limited and is only located at the side closest to the center axis of the resulting particle. This is not the case for blunt particle ends, in which several nanorods are most likely fused together in parallel, resulting in much higher number of internal Al sites along their full length.

The correlative nano-imaging shows that catalytic hotspots are related to crystal imperfections that locally enhance active site accessibility. Al sites can only optimally fulfill their catalytic role if both reagent and product molecules can easily diffuse through the micropores containing the active sites. In case of the H-ZSM-22 framework, only the 10 membered ring (10-MR) channels allow sufficient mass transport for catalysis purposes. This limits diffusion through the micropores, possibly hampering catalytic activity. However, an imperfect fusion between the elementary nanorods could leave a gap in the catalyst structure and decrease the average distance between the micropore entrances and the active sites, thus increasing 
mass transport. A similar explanation holds for the nanorod fused on top of the needle-shaped particle in Figure 2J. An incomplete fusion with the underlying particle might lead to additional openings into the catalyst structure, making the internal Al more accessible. The current ILEM-based research provides a direct correlation of catalytic hotspots to the presence of structural defects, such as incomplete intergrowths. It must be noted, however, that not all catalytic hotspots in the investigated $\mathrm{H}-\mathrm{ZSM}-22$ particles can be related to readily visible structural features. Besides defects present at the bottom side of the crystal, which will not be resolvable by SEM there is also still room for improving the resolution as very fine structural features remain unresolved. Care however must be taken to minimize sample damage under electron beam irradiation. Previous research has demonstrated that prolonged electron beam exposure can induce chemical and structural changes to zeolites and molecular sieve catalysts. ${ }^{[34-36]}$ Rationally, such electron beam induced amorphisation would impact the catalytic activity and seems to be most precarious in small, nanometer sized zeolite particles. In this regard, the sample under investigation and, more specifically, the outer edges and the sharp particle tips, appear particularly prone to electron beam damage as these are the narrowest structural features. This is confirmed by an additional experiment in which several H-ZSM-22 particles are exposed to at least 5-fold more intense electron beam irradiation (Half field-of-view (FOV) scanned in $2 \mathrm{~min}, 5 \mathrm{keV}$ acceleration voltage, $0.19 \mathrm{nA}$ ) and one particle within the same FOV is only irradiated using the mild imaging conditions used throughout this work (full FOV scanned once in $1.4 \mathrm{~min}, 2 \mathrm{keV}$ acceleration voltage, $0.10 \mathrm{nA}$ ). The result of this experiment is shown in Figure $\mathbf{S 6}$ and reveals that the catalyst particles become catalytically inactive after intense electron beam irradiation, however, the mildly irradiated catalyst particle still shows activity.

Clearly, the effect of the SEM imaging conditions applied during the presented research on the sample integrity needs to be carefully considered. In order to double check the previously discussed results obtained with the mild electron doses, the correlated experiments were performed in a reversed sequence, i.e. the SEM image is acquired after performing the chemical activity mapping (Figure S7). Performing the experiments in a reversed order is, however, not the method of choice, as the removal of the liquid after the catalytic activity measurements enhances the risk of unwanted sample movement; small shifts hamper correct image correlation. Further, after removal of the liquid a residual film might remain on the sample that reduces the amount of detail that can be resolved in the SEM images (Figure S7F-G). The results of four validation experiments that were performed reveal a similar reactivity distribution compared to the previous experiments using the initial experimental approach (Figure 2). Hence, these results demonstrate that the electron beam effect, at least under the mild SEM imaging conditions used in this work (2 keV and $0.15 \mathrm{nA}$ ), on the chemical reactivity in the described $\mathrm{H}-\mathrm{ZSM}$ 22 investigation is negligible. Using such milder imaging conditions additionally reduces sample charging, which is required as the sample is mounted on insulating glass cover slides.

To further confirm the importance of nanorod fusion in generating catalytically active crystal domains, the molecular confinement of the observed catalytic reaction products to the one-dimensional pore network of $\mathrm{H}-\mathrm{ZSM}-22$ was evaluated. Specifically for H-ZSM-22, the presence of acid sites at the exterior zeolite surface would have a negative impact in various processes. In n-alkane hydroisomerization, for example, this would deteriorate catalyst shape selectivity. ${ }^{[37,38]}$ The molecular confinement of the individual fluorescent reaction products with respect to the $\mathrm{H}-\mathrm{ZSM}-22$ crystals and the underlying pore structure was investigated using linear polarized excitation light. Note that the fluorescent FFA oligomers will only be efficiently excited when its dipole moment is oriented parallel to the polarization of the excitation light. The dimensions of the FFA oligomers are such that their long axis needs to be aligned along the 10-MR pores. The results of such a linear polarized excitation light experiment on a representative $\mathrm{H}-\mathrm{ZSM}-22$ particle are shown in Figure 3.

When mapping the catalytic activity distribution using linear polarized excitation light along the $10-\mathrm{MR}$ pores (Figure $3 \mathrm{~A}$ ), a similar reactivity is observed as when using circular polarized excitation light (Figure 2). In both cases a heterogeneous activity profile is revealed along the long axis of the particle and the edges and sharp tips of the particle are completely inactive. The activity map obtained after changing the polarization direction with 90 degrees (Figure $3 \mathrm{~B}$ ) results in a complete disappearance of the fluorescent reaction products. This shows that all fluorescent product molecules are confined to the microporous structure. Based on the results shown in Figure 2 and 3 , the overall activity of $\mathrm{H}-\mathrm{ZSM}-22$ particles can be schematically represented as in Figure 4. Hence, it is demonstrated beyond doubt that catalytic transformations of FFA exclusively occur on acid sites contained inside the
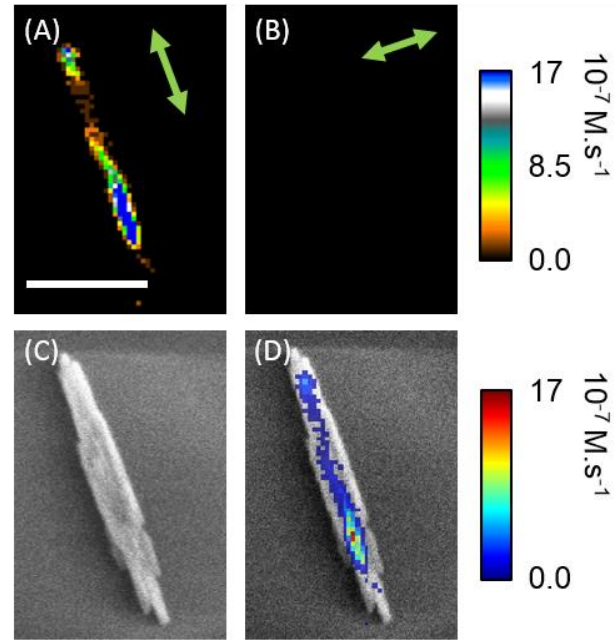

microporous structure, leading to the highly shape-selective properties.

Figure 3. (A) Catalytic activity map obtained with linear polarized excitation light along and (B) perpendicular to the one dimensional porous structure in a needle-shaped $\mathrm{H}-\mathrm{ZSM}-22$ particle. The green double headed arrows indicate the 
respective light polarization direction and the color scale represents the reactivity within the individual bins. (C) The corresponding SEM image of the particle under investigation and (D) the correlative micrograph in combination with a color scale that qualitatively indicates reactivity differences in the different areas of the catalyst particle. (Scale bar $=500 \mathrm{~nm}$ ).

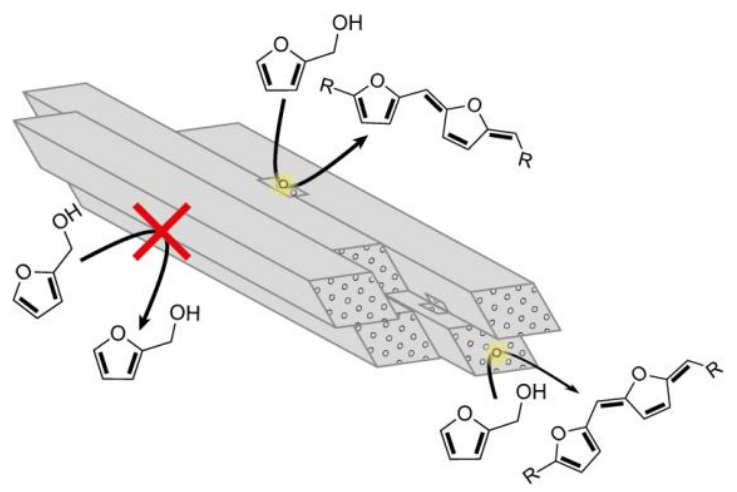

Figure 4. Schematic representation of the catalytic activity distribution of needle-shaped H-ZSM-22 crystals. Internal aluminum results in catalytically active acid sites that are located within the microporous structure. These active sites are accessible through the 10-MR pore entrances (dotted pattern) that are present on the tips of the individual nanorods, or at crystallographic defects that originate from imperfect lateral nanorod fusion. Framework aluminum at the external surface are catalytically inactive.

\section{Conclusion}

Correlative super-resolution fluorescence and scanning electron microscopy has been used to investigate the catalytic activity of individual H-ZSM-22 catalyst particles. This has, for the first time, directly revealed that catalytic activity is confined to the inner parts of needle-shaped H-ZSM-22 crystals where the catalytically inactive external Al, present at the outer edges of the primary nanorods, is converted into catalytically active internal Al. As a result, the outer shell of the crystals, with a thickness of $20-40 \mathrm{~nm}$, is catalytically inactive. This further adds to the shape selectivity imposed by the 10-MR pore dimensions, by avoiding undesirable product formation at the external surface of the zeolite particles. Additionally, catalytic hotspots are prominently present in each crystal. Based on the correlated SEM images these could be assigned to crystal defects such as mismatching during lateral nanorod fusion. However, variations in Al distribution at these scales cannot be ruled out due to a lack of sufficiently accurate experimental methods. The confinement of the catalytic process to the 10-MR micropores could further be confirmed from the linear polarized excitation experiments. These support that catalytic activity is confined to Al sites present within the internal surface of the microporous structure. Future efforts should focus on investigating the precise interplay between structural imperfections and improved catalytic activity. For example, the development of tools that combine high resolution-SEM or -TEM with NASCA microscopy would enable such research, for example on the basis of already existing systems combining TEM and laserscanning fluorescence microscopy. ${ }^{[39,40]}$

\section{Conflicts of interest}

There are no conflicts to declare.

\section{Acknowledgements}

The authors thank "Fonds voor Wetenschappelijk Onderzoek" (Grant G0962.13, AKUL/11/14), KU Leuven Research Fund (C14/15/053). A.V.K. is a Postdoctoral Fellow of the Research Foundation - Flanders (FWO), project number 12 Y $3719 \mathrm{~N}$. The research leading to these results has received funding from the European Research Council under the European Union's Seventh Framework Programme (FP/2007-2013)/ERC Grant Agreement no. [307523], ERC-Stg LIGHT to M.B.J.R. We sincerely thank Professor Sara Bals and Mr. Kadir Sentosun at EMAT at University of Antwerp for helping us with HR-TEM and electron diffraction measurements.

\section{Notes and references}

[1] N. Mizuno, M. Misono, Chem. Rev. 1998, 98, 199-218.

[2] J. Weitkamp, Solid State lonics 2000, 131, 175-188.

[3] A. Corma, J. Catal. 2003, 216, 298-312.

[4] A. Bhan, E. Iglesia, Acc. Chem. Res. 2008, 41, 559-567.

[5] C. Baerlocher, L. B. McCusker, D. Olson, W. M. Meier, Atlas of Zeolite Framework Types, Published On Behalf Of The Structure Commission Of The International Zeolite Association By Elsevier, 2007.

[6] N. Danilina, F. Krumeich, S. A. Castelanelli, J. A. van Bokhoven, J. Phys. Chem. C 2010, 114, 6640-6645.

[7] J. Dědeček, Z. Sobalík, B. Wichterlová, Catal. Rev. 2012, 54, 135-223.

[8] G. T. Kokotailo, J. L. Schlenker, F. G. Dwyer, E. W. Valyocsik, Zeolites 1985, 5, 349-351.

[9] R. Kumar, P. Ratnasamy, J. Catal. 1989, 116, 440-448.

[10] S. Teketel, W. Skistad, S. Benard, U. Olsbye, K. P. Lillerud, P. Beato, S. Svelle, ACS Catal. 2012, 2, 26-37.

[11] Z.-M. Cui, Q. Liu, Z. Ma, S.-W. Bian, W.-G. Song, J. Catal. 2008, 258, 83-86.

[12] D. Verboekend, K. Thomas, M. Milina, S. Mitchell, J. PérezRamírez, J.-P. Gilson, Catal. Sci. Technol. 2011, 1, 13311335.

[13] K. Hayasaka, D. Liang, W. Huybrechts, B. R. De Waele, K. J. Houthoofd, P. Eloy, E. M. Gaigneaux, G. van Tendeloo, J. W. Thybaut, G. B. Marin, et al., Chem. - A Eur. J. 2007, 13, 10070-10077.

[14] D. Verboekend, A. M. Chabaneix, K. Thomas, J.-P. Gilson, J. Pérez-Ramírez, CrystEngComm 2011, 13, 3408-3416.

[15] A. W. Chester, W. E. Garwood, J. C. Vartuli, A. W. Chester W. E. Garwood, J. C. Vartuli, US 4605488, 1986. 
[16] S. I. Hommeltoft, S. J. Miller, A. Pradhan, S. I. Hommeltoft, S. J. Miller, A. Pradhan, US 4717465, 2011.

[17] J. A. Martens, D. Verboekend, K. Thomas, G. Vanbutsele, J.P. Gilson, J. Pérez-Ramírez, ChemSusChem 2013, 6, 421425.

[18] T. L. M. Maesen, M. Schenk, T. J. H. Vlugt, J. P. d. Jonge, B. Smit, J. Catal. 1999, 188, 403-412.

[19] J. A. Martens, W. Souverijns, W. Verrelst, R. Parton, G. F. Froment, P. A. Jacobs, Angew. Chemie Int. Ed. English 1995, 34, 2528-2530.

[20] A. K. Jamil, O. Muraza, M. Sanhoob, T. Tago, H. Konno, Y. Nakasaka, T. Masuda, J. Anal. Appl. Pyrolysis 2014, 110, 338-345.

[21] A. K. Jamil, O. Muraza, M. Yoshioka, A. M. Al-Amer, Z. H. Yamani, T. Yokoi, Ind. Eng. Chem. Res. 2014, 53, 1949819505.

[22] S. Teketel, S. Svelle, K.-P. Lillerud, U. Olsbye, ChemCatChem 2009, 1, 78-81.

[23] T. Q. Hoang, X. Zhu, T. Danuthai, L. L. Lobban, D. E. Resasco, R. G. Mallinson, Energy \& Fuels 2010, 24, 38043809.

[24] Y. Luo, Z. Wang, S. Jin, B. Zhang, H. Sun, X. Yuan, W. Yang, CrystEngComm 2016, 18, 5611-5615.

[25] F. Di Renzo, F. Remoué, P. Massiani, F. Fajula, F. Figueras, C. Thierry Des, Zeolites 1991, 11, 539-548.

[26] J. Čejka, A. Corma, S. Zones, Eds. , Zeolites and Catalysis : Synthesis, Reactions and Applications, Wiley-VCH Verlag $\mathrm{GmbH}$ \& Co. KGaA, 2010.

[27] B. M. Weckhuysen, Angew. Chemie - Int. Ed. 2009, 48, 4910-4943.

[28] I. L. C. Buurmans, B. M. Weckhuysen, Nat. Chem. 2012, 4, 873-886.

[29] K. P. F. Janssen, G. De Cremer, R. K. Neely, A. V. Kubarev, J. Van Loon, J. A. Martens, D. E. De Vos, M. B. J. Roeffaers, J. Hofkens, Chem. Soc. Rev. 2014, 43, 990-1006.

[30] M. B. J. Roeffaers, G. De Cremer, H. Uji-i, B. Muls, B. F. Sels, P. A. Jacobs, F. C. De Schryver, D. E. De Vos, J. Hofkens, Proc. Natl. Acad. Sci. U.S.A. 2007, 104, 12603-12609.

[31] W. Xu, J. S. Kong, Y.-T. E. Yeh, P. Chen, Nat. Mater. 2008, 7, 992-996.

[32] M. B. J. Roeffaers, G. De Cremer, J. Libeert, R. Ameloot, P. Dedecker, A.-J. Bons, M. Bückins, J. A. Martens, B. F. Sels, D. E. De Vos, et al., Angew. Chemie - Int. Ed. 2009, 121, 9285-9289.

[33] X. Zhou, N. M. Andoy, G. Liu, E. Choudhary, K.-S. S. Han, H. Shen, P. Chen, Nat. Nanotechnol. 2012, 7, 237-241.

[34] L. A. Bursill, J. M. Thomas, K. J. Rao, Nature 1981, 289, 157158.

[35] E. H. Hirsch, Nature 1981, 293, 759-759.

[36] C. Aydin, J. Lu, A. J. Liang, C.-Y. Chen, N. D. Browning, B. C. Gates, Nano Lett. 2011, 11, 5537-5541.

[37] N. Chen, N. Wang, Y. Ren, H. Tominaga, E. W. Qian, J. Catal. 2017, 345, 124-134.

[38] J. A. Martens, G. Vanbutsele, P. A. Jacobs, J. Denayer, R. Ocakoglu, G. Baron, J. A. Muñoz Arroyo, J. Thybaut, G. B. Marin, Catal. Today 2001, 65, 111-116.

[39] M. A. Karreman, I. L. C. Buurmans, A. V. Agronskaia, J. W.
Geus, H. C. Gerritsen, B. M. Weckhuysen, Chem. - A Eur. J. 2013, 19, 3846-3859.

[40] M. A. Karreman, I. L. C. Buurmans, J. W. Geus, A. V. Agronskaia, J. Ruiz-Martínez, H. C. Gerritsen, B. M. Weckhuysen, Angew. Chemie Int. Ed. 2012, 51, 1428-1431. 